\title{
ATLAS prospects for beyond the Standard Model searches
}

\author{
F. Ledroit-Guillon ${ }^{\mathrm{a}}$, on behalf of the ATLAS collaboration \\ ${ }^{a}$ LPSC, 53 rue des Martyrs, 38026 Grenoble Cedex, France
}

We discuss how ATLAS has been preparing for the analysis of the first $\mathrm{fb}^{-1}$ of good data at $14 \mathrm{TeV}$ in view of discovering new physics beyond the Standard Model. We show some ideas developed for understanding the backgrounds and we present as realistic as possible estimates of the reach of the experiment.

\section{INTRODUCTION}

It is well understood today that the Standard Model is not the ultimate theory, and that we have to go beyond. A number of theoretical models has thus been proposed, the most popular so far being supersymmetry (SUSY). Several alternatives to supersymmetry have nevertheless been developed, including Grand Unified Theories, theories with extra dimensions, etc. This paper reviews some of the most salient results from the prospective studies performed by the ATLAS collaboration on the discovery potential of SUSY and alternatives (called "Exotics" in ATLAS). Only results which were public at the time of the conference are shown, thus based on Monte Carlo simulations at the design centre of mass en$\mathrm{ergy}^{1}$ of $14 \mathrm{TeV}$. Furthermore, the results in general assume an initial data sample of about $1 \mathrm{fb}^{-1}$ of integrated luminosity.

\section{THE ATLAS DETECTOR}

The ATLAS detector is a general multi-purpose detector which is described in detail in [1]. It comprises an Inner Detector which combines silicon (pixels and strips) and transition radiation trackers, with full coverage up to a pseudorapidity $|\eta|$ of 2.5. The tracker is surrounded by a $2 \mathrm{~T}$ solenoid. Beyond the coil, there is a complete calorimetric system with electromagnetic (with an expected resolution of $\sigma / E \simeq 10 \% / \sqrt{E} \oplus$

\footnotetext{
${ }^{1}$ In some cases, the centre of mass energy has the value which was expected at the time of the conference for the first physics run, namely $10 \mathrm{TeV}$.
}

$0.7 \%)$, hadronic and low angle calorimeters. Finally, one finds a Muon Spectrometer based on powerful air-core toroids and muon chambers which together provide a transverse momentum resolution $\sigma / p_{T}$ of about $10 \%$ at $1 \mathrm{TeV}$.

During the 2008-2009 period, ATLAS has recorded more than 200 million cosmic ray events, as well as randomly triggered events, in various configurations. After analyzing these data, we know that ATLAS will enter the 2009-2010 data taking period with very few dead channels (of the order of 1\%) and a low and well understood level of noise. As an illustration, the performance on missing transverse energy $\left(E_{T}^{\text {miss }}\right)$ has been evaluated by means of random triggers. The energy of all the calorimeter (electromagnetic and hadronic) cells with an energy above a noise threshold of two standard deviations has been summed and the following quantity computed:

$$
E_{T}^{\text {miss }}=\sqrt{\left(E_{X}^{m i s s}\right)^{2}+\left(E_{Y}^{m i s s}\right)^{2}}
$$

with $E_{X}^{\text {miss }}=-\Sigma E \sin \theta \cos \phi$ and $E_{Y}^{\text {miss }}=$ $-\Sigma E \sin \theta \sin \phi$. Figure 1 shows the distribution of $E_{T}^{\text {miss }}$; since no real energy is expected to be deposited, the only contribution to the missing transverse energy is electronic noise. Figure 1 also shows the distribution of the same observable computed with clusters rather than cells. The cell-derived distribution characterizes the basic calorimeter performance, whereas the cluster derived one is close to the missing transverse energy reconstruction algorithm that will be used for the analysis of collision data. The results show a good control of the energy reconstruction in 


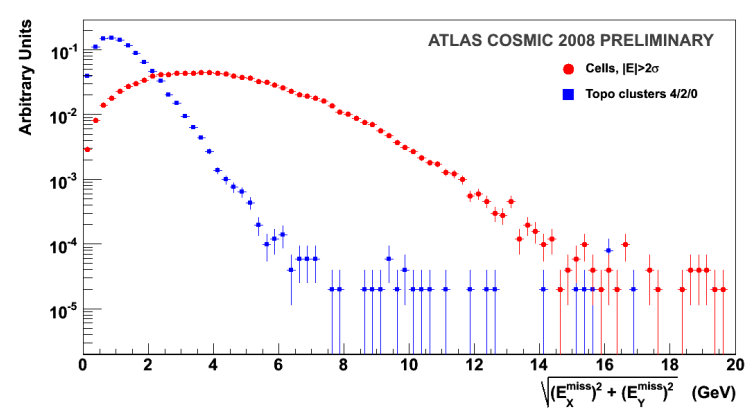

Figure 1. Inclusive distributions of $E_{T}^{\text {miss }}$ for randomly triggered events.

the 187000 cells of the calorimeter. The clusterbased distribution shows a better noise suppression. Tails in the distributions (beyond $8 \mathrm{GeV}$ for the cluster-based, and $16 \mathrm{GeV}$ in the cellbased variable), contributing less than $0.1 \%$ of events, have been understood to come from coherent noise in a specific region of the calorimeter, due to a now replaced faulty device. This illustrates the good shape of the ATLAS detector for $E_{T}^{m i s s}$ reconstruction, a crucial observable in most SUSY searches.

\section{SUPERSYMMETRY}

At the LHC, the production of sparticles is dominated by strongly interacting particles, namely squarks and gluinos. Therefore, even though supersymmetry comes in many flavours depending on its breaking mechanism (minimal SUGRA, GMSB, AMSB, split SUSY,...) as soon as we assume that R-parity is conserved, there is a common topology for most SUSY events which is the following: high transverse energy jets, coming from the decays of the squarks or gluinos, leptons, from the decays of the subsequent gauginos, and missing transverse energy from the escape of the lightest supersymmetric particle (LSP). The production cross sections then primarily depend on the masses.

The strategy of ATLAS is therefore to design analyses which are sensitive to inclusive topologies of this type, the idea being to maximise the coverage of the search independently of the exact value of the numerous parameters of the model. The minimal SUGRA model is then used as a benchmark in the design of the analysis, and inside this model, several benchmark points labeled SUn are studied, spanning a broad parameter space addressing very different final state topologies - see table 1 for the parameter values.

Table 1

Parameters of SUSY benchmark points.

\begin{tabular}{lrrrr}
\hline Point & $\begin{array}{r}m_{0} \\
(\mathrm{GeV})\end{array}$ & $\begin{array}{r}m_{1 / 2} \\
(\mathrm{GeV})\end{array}$ & $\begin{array}{r}A_{0} \\
(\mathrm{GeV})\end{array}$ & $\tan \beta$ \\
\hline SU1 & 70 & 350 & 0 & 10 \\
SU2 & 3550 & 300 & 0 & 10 \\
SU3 & 100 & 300 & -300 & 6 \\
SU4 & 200 & 160 & -400 & 10 \\
SU6 & 320 & 375 & 0 & 50 \\
SU8.1 & 210 & 360 & 0 & 40 \\
\hline
\end{tabular}

All points have $\mu>0$.

For selecting SUSY events, a number of powerful observables have been designed in addition to the missing transverse energy: mainly the effective mass $M_{\text {eff }}$, the transverse sphericity $\left(S_{T}\right)$ and the transverse mass $M_{T}$.

$$
M_{\text {eff }}=\sum_{i=1}^{4} p_{T}^{j e t, i}+E_{T}^{m i s s}\left[+\sum_{i=1} p_{T}^{l e p, i}\right]
$$

where the sums run respectively over the four highest $p_{T}$ jets within $|\eta|<2.5$ and over all the identified leptons.

$$
S_{T}=\frac{2 \lambda_{2}}{\lambda_{1}+\lambda_{2}}
$$

where $\lambda_{1}$ and $\lambda_{2}$ are the eigenvalues of the $2 \times 2$ sphericity tensor $S_{i j}=\sum_{k} p_{k i} p^{k j}$.

$M_{T}^{2}\left(\mathbf{p}_{T}^{\alpha}, \mathbf{p}_{T}^{m i s s}, m_{\alpha}\right)=m_{\alpha}^{2}+2\left(E_{T}^{\alpha} E_{T}^{m i s s}-\mathbf{p}_{T}^{\alpha} \cdot \mathbf{p}_{T}^{m i s s}\right)$,

where $E_{T}^{\alpha}=\sqrt{\left(\mathbf{p}_{T}^{\alpha}\right)^{2}+m_{\alpha}^{2}}, E_{T}^{\text {miss }}=\sqrt{\left(\mathbf{p}_{T}^{\text {miss }}\right)^{2}}$ and $\alpha$ is some visible particle.

The studies in [2] applied the following baseline event selection: at least 4 jets of at least 
$50 \mathrm{GeV}$ transverse energy (at least one jet must have $\left.p_{T}>100 \mathrm{GeV}\right) ; E_{T}^{\text {miss }}>100 \mathrm{GeV} ; E_{T}^{\text {miss }}>$ $0.2 M_{\text {eff }}$ (this is against Gaussian fluctuations of the $E_{T}^{\text {miss }}$ measurement); $S_{T}>0.2$ (this selection is efficient against QCD background); exactly 0 or 1 or 2 identified leptons (electrons or muons). Additional criteria are, in the no lepton case: the angle in the transverse plane between the three most energetic jets and the missing transverse energy $\left(\Delta \phi\left(j, E_{T}^{m i s s}\right)\right)$ must be greater than 0.2 ; in the 1 or 2 lepton case, the transverse mass of any lepton and the missing transverse energy must be greater than $100 \mathrm{GeV}$. Lower multiplicity topologies requiring a lower number of QCD jets were also studied.

Indeed, the backgrounds to fight are, on the one hand, the QCD multijet events with their huge cross section, especially relevant in the no-lepton case, in which instrumental effects can fake missing energy. The $\Delta \phi\left(j, E_{T}^{\text {miss }}\right)$ selection is meant to reduce this type of background. On the other hand, top quark pairs, and $\mathrm{W}$ and $\mathrm{Z}$ production with additional jets events, although they have lower cross sections than QCD, yield final states with real missing transverse energy through the decay into real neutrinos. In this case, it is the transverse mass selection which is used to lower the background level.

In the year preceding the beginning of data taking, ATLAS has put a strong emphasis on studies meant to ensure a good control of the backgrounds and has in particular explored several methods allowing their estimation from the data themselves. Examples of such methods will be given in the following. All results presented are described in detail in [2].

\subsection{Inclusive zero lepton analysis}

Figure 2 shows, for $1 \mathrm{fb}^{-1}$ of well understood data, the effective mass distribution for each benchmark point and for the sum of all standard model backgrounds after the baseline selection with a lepton veto. There is clearly a very high sensitivity for all points, except the one labeled SU2 for which the cross section is dominated by direct gaugino production. The components of the background can be seen on figure 3 .

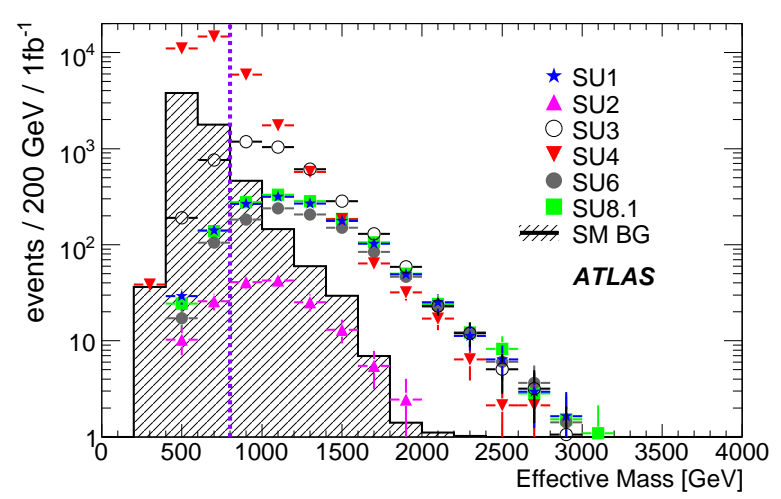

Figure 2. $M_{\text {eff }}$ distribution for all SUSY benchmark points and the total Standard Model background after the full baseline selection with a lepton veto.

\section{QCD multijet background}

For the early phase of data taking, data driven estimates of QCD backgrounds, which are difficult to simulate reliably, will be a priority. A possible method for this purpose consists in smearing jet transverse momenta in the low $E_{T}^{\text {miss }}$ region with a data-measured jet response function $R$ defined as the event-by-event ratio of measured jet $p_{T}$ to true jet $p_{T}$.

In a first step, one measures the Gaussian response function of ATLAS calorimeters to jets, making use of the balance in $\gamma+$ jet events. Because of the limited statistics of the $\gamma+$ jet sample, a second step is required in order to measure the full (Gaussian plus non Gaussian) response function. Here, the idea is to use events in which the $E_{T}^{\text {miss }}$ vector can be unambiguously associated in $\phi$ with a single jet. This is achieved by selecting events with three high $p_{T}$ jets well separated in $\phi$. The response to jet $J$ can then be obtained from

$$
R=\frac{\mathbf{p}_{T}(J) \cdot \mathbf{p}_{T}(J, \text { true })}{\mid\left.\mathbf{p}_{T}(J, \text { true })\right|^{2}} .
$$

Assuming that $E_{T}^{m i s s}$ in these events is dominated by fluctuations in $\mathbf{p}_{T}$, one can then approximate $\mathbf{p}_{T}(J$, true $) \simeq \mathbf{p}_{T}(J)+\mathbf{p}_{T}^{\text {miss }}$. The resulting $R$ distribution is shown in figure 4 , with a normalization obtained from the study of the response 


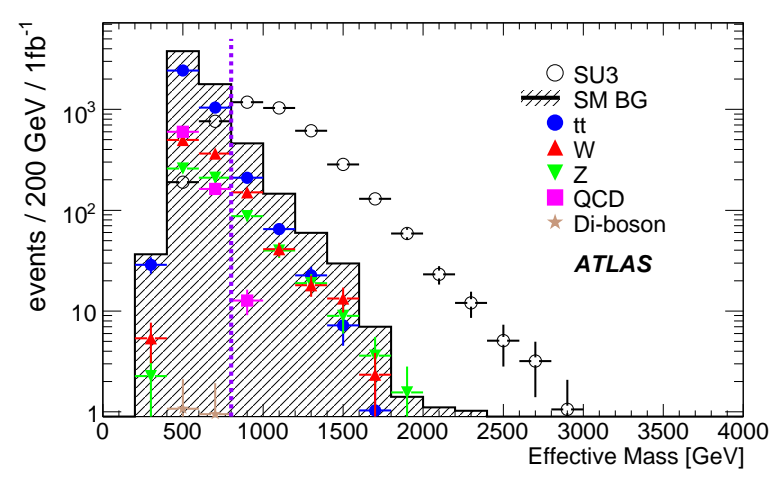

Figure 3. $M_{\text {eff }}$ distribution for the Standard Model backgrounds and one SUSY benchmark point after the full baseline selection with a lepton veto.

using dijet events.

In a third and last step, a control sample of multijet events with low $E_{T}^{m i s s}$ is used to estimate the $E_{T}^{m i s s}$ and $M_{\text {eff }}$ distributions of QCD multijet events by smearing the jet transverse momenta with the response function $R$ measured in step 2. A good agreement between the estimated $E_{T}^{m i s s}$ and $M_{\text {eff }}$ distributions and the ones directly obtained from GEANT4 is obtained before and after applying the full selection.

\section{$Z \rightarrow \nu \bar{\nu}$ background}

The $Z \rightarrow \nu \bar{\nu}$ background is another one of the main background processes in the no-lepton channel. In order to estimate and reproduce the number of expected background events, as well as the shape of the $E_{T}^{\text {miss }}$ and $M_{\text {eff }}$ distributions, $Z \rightarrow \ell^{+} \ell^{-}$events $(\ell=e, \mu)$ are selected, in which the charged leptons are replaced by neutrinos. The events are selected with the same criteria (except for the leptons) with the $E_{T}^{\text {miss }}$ selection replaced by $p_{T}\left(\ell^{+} \ell^{-}\right) \simeq p_{T}(Z)$. In order to increase the statistics, also events with one low quality electron (" $X$ ") are used. A number of corrections are then applied:

- a fiducial correction $\left(c_{F i d u}\right)$ due to the lack of lepton identification beyond $|\eta|=2.5$;

- a kinematics correction $\left(c_{K i n}\right)$ for the addi-

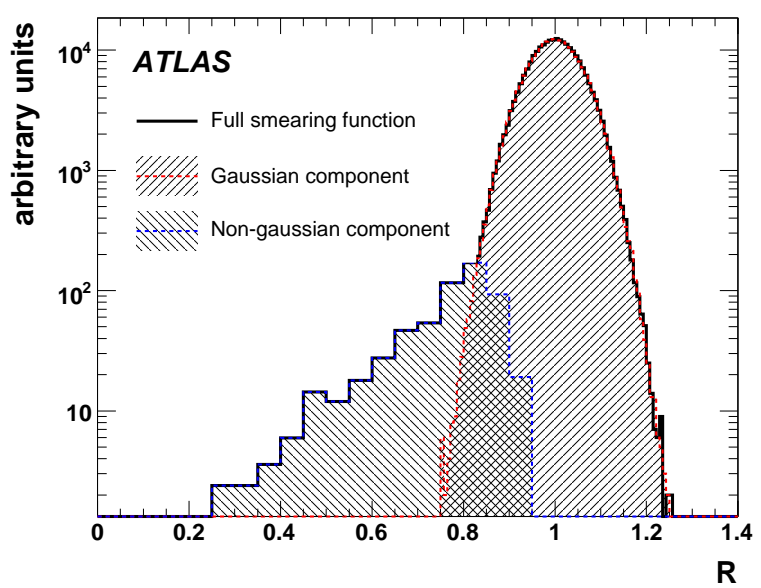

Figure 4. Smearing function for a jet of $250 \mathrm{GeV}$ (thick line) with Gaussian and non-Gaussian components shown separately.

tional cuts used to select $Z \rightarrow \ell^{+} \ell^{-}$events;

- a correction for the lepton identification efficiency (included in $c_{F i d u}$ ).

In each $E_{T}^{m i s s}$ bin:

$$
\begin{gathered}
N_{Z \rightarrow \nu \bar{\nu}}\left(E_{T}^{\text {miss }}\right)=N_{Z \rightarrow \ell^{+} \ell^{-}}\left(p_{T}\left(\ell^{+} \ell^{-}\right)\right) \\
\times c_{\text {Kin }}\left(p_{T}(Z)\right) \times c_{\text {Fidu }}\left(p_{T}(Z)\right) \times \frac{\operatorname{Br}(Z \rightarrow \nu \bar{\nu})}{\operatorname{Br}\left(Z \rightarrow \ell^{+} \ell^{-}\right)}
\end{gathered}
$$

Figure 5 shows a good agreement between the $E_{T}^{m i s s}$ distributions of $Z \rightarrow \nu \bar{\nu}$ and corrected $Z \rightarrow$ $\ell^{+} \ell^{-}$events; however, some parameterization will be needed to extrapolate at very high values of $E_{T}^{m i s s}$ because the statistics is low in this regime.

\subsection{Inclusive one lepton analysis}

In this search mode, the presence of a high $p_{T}$ isolated electron or muon suppresses the QCD background, but also costs signal efficiency. Nevertheless, it is a robust way to look for SUSY and it will play an important role. Figure 6, as previously for the no-lepton mode, shows the effective mass distribution for various benchmark points and for the sum of all standard model backgrounds, dominated by $t \bar{t}$, after the baseline selection with a one lepton requirement. Again, there 


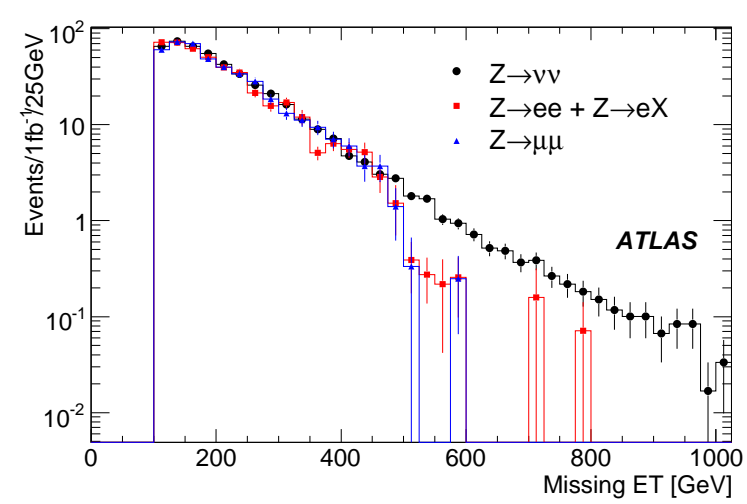

Figure 5. $E_{T}^{\text {miss }}$ distribution after all corrections for $Z \rightarrow \nu \bar{\nu}, Z \rightarrow e^{+} e^{-}+e^{ \pm} X$ and $Z \rightarrow \mu^{+} \mu^{-}$ processes. The number of events corresponds to an integrated luminosity of $1 \mathrm{fb}^{-1}$.

is clearly a very high sensitivity for most SUSY points.

\section{Top and $\mathrm{W}$ plus jets background}

Many methods have been studied by ATLAS for estimating the $\mathrm{W}$ and top pairs backgrounds from the data. One example of such methods is the creation of a control sample by reversing the selection on the transverse mass $M_{T}$, which discriminates very effectively the top and $\mathrm{W}^{ \pm}$. After checking that $M_{T}$ only weakly depends on $E_{T}^{\text {miss }}$, one can select events with small $M_{T}$ (typically less than $100 \mathrm{GeV}$, the control region) in which the $t \bar{t}$ and $\mathrm{W}^{ \pm}$processes are enhanced over other processes, including SUSY, while the large $M_{T}$ sample is the signal region (see figure 7). The expected background events in the signal region is then extrapolated from the control region, the relative normalization being derived from the low $E_{T}^{\text {miss }}$ or $M_{\text {eff }}$ regions: $N(B)=N(D) \times N(A) / N(C)$, where $N(X)$ is the number of background events in region $X$ (see figure 8). This works very well as long as there is no SUSY signal in the control regions. However, if sparticles are produced, they are likely to contribute also to the control samples, leading to an overestimation of the background. It has been checked that for the studied benchmark points,

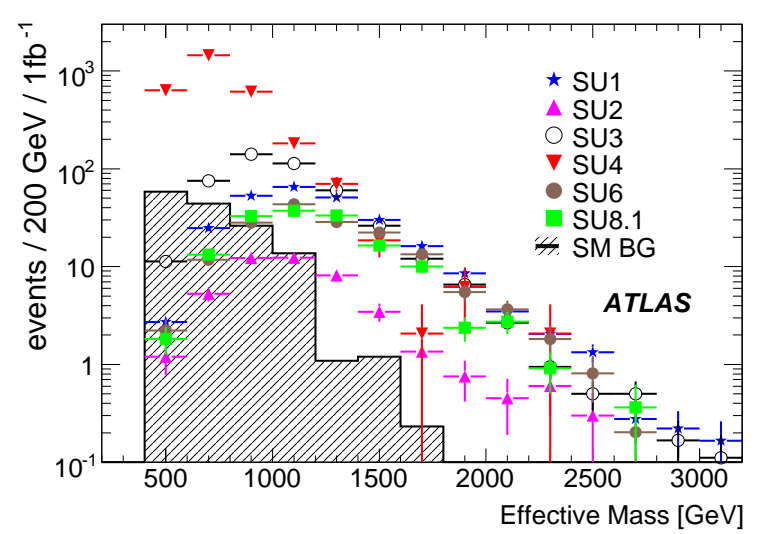

Figure 6. The $M_{\text {eff }}$ distributions for each of the benchmark points and for the sum of the Standard Model backgrounds with $1 \mathrm{fb}^{-1}$ for the 1 lepton analysis after all cuts.

the amount of over-estimation is smaller than the signal itself, and thus a clear excess can still be observed. Nevertheless, one can also try and find a way to improve the background estimation, even in presence of supersymmetric background. ATLAS has for instance experimented an iterative procedure based on the shape of the $M_{T}$ distribution which gives good results (see ref. [3]). This field is very active currently in the ATLAS collaboration.

\subsection{Inclusive analyses discovery reach}

In addition to the zero- and one-lepton analyses described above, ATLAS has studied several other inclusive topologies : two-lepton (same charge or opposite charge) trileptons, tau-leptons, b-jets. In the same way, many more background studies have been performed (which are also described in [2]). Their combination allows to compute the total systematic uncertainty expected from the background estimation. Assuming $1 \mathrm{fb}^{-1}$ of well understood data, it is expected to be around $50 \%$ for QCD and $20 \%$ for $\mathrm{W} / \mathrm{Z} /$ top processes.

In order to evaluate the discovery potential, the significance of the signal is calculated from the probability of the background, including uncer- 


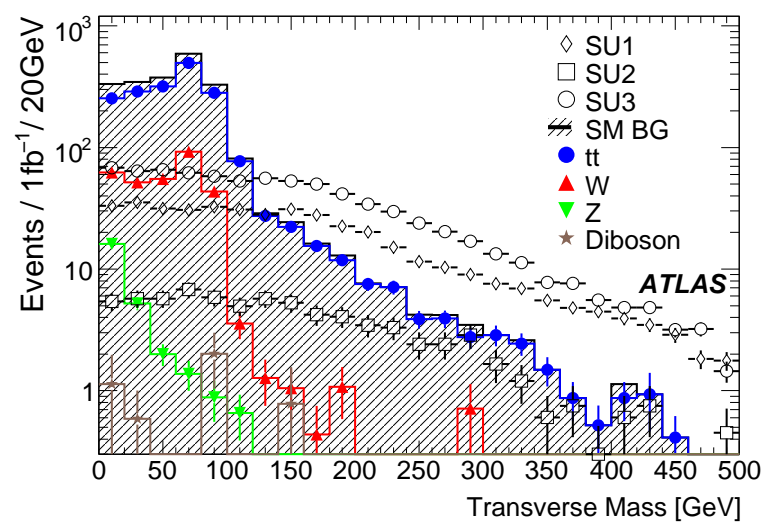

Figure 7. Transverse mass distribution of various SUSY signals. Background processes are superimposed for comparison; the hatched histogram shows the sum of all Standard Model backgrounds.

tainties, to fluctuate to the signal. A scan of the mSUGRA parameter space is performed, using leading order cross sections and fast (parameterized) simulation. In addition to the baseline selections described above, a selection on the effective mass is applied, which is optimized for each point of the scan to yield the best significance. A typical value for the $M_{\text {eff }}$ cut for SUSY masses in the $\mathrm{TeV}$ region is around $800 \mathrm{GeV}$. The trigger ${ }^{2}$ is included in the efficiency calculation. The resulting $5 \sigma$ coverage of the $\left(m_{0}, m_{1 / 2}\right)$ plane is shown for instance for $\tan \beta=10$ in figure 9 . The reach is dominated by the $E_{T}^{\text {miss }}+$ jets (no-lepton) analysis, but many other signatures (not all described here) cover most of the phase space. A scan assuming a large value of $\tan \beta$ has shown a very good coverage of the plane as well.

\subsection{Final states with photons and long lived charged particles}

In models with gauge-mediated SUSY breaking (GMSB), the LSP is a gravitino and can be very light. The next-to-lightest SUSY particle (NLSP)

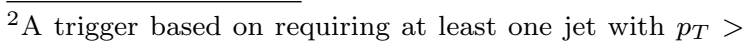
$70 \mathrm{GeV}$ plus $E_{T}^{\text {miss }}>70 \mathrm{GeV}$ has an efficiency better than $97 \%$ for all benchmark points.
}

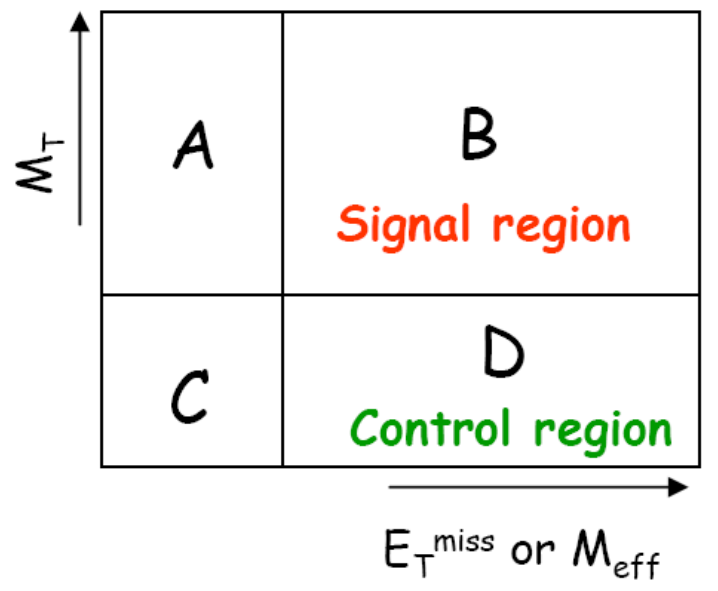

Figure 8. Definition of signal and control regions.

is typically either the lightest neutralino or a slepton, depending on the number of SU(5) messenger multiplets $\left(N_{5}\right)$. The NLSP decays into the LSP plus typically a photon or a lepton. This decay can be prompt or the NLSP can have a significant lifetime, depending on the value of the gravitino coupling to the LSP $\left(C_{\text {Grav }}\right)$.

ATLAS has used the same baseline preselection as above (without the $S_{T}$ selection), complemented by the requirement of at least one isolated photon to evaluate the discovery potential of SUSY assuming a GMSB model with the lightest neutralino NLSP. Again, scanning the parameter space with the help of fast simulation, the discovery potential in the $(\Lambda, \tan \beta)$ plane shown in figure 10 has been obtained in the prompt photon scenario. On the other hand, it is to be noted that in the $C_{\text {Grav }}>1$ case which leads to non pointing photon topologies, it could be possible to determine the neutralino lifetime. More details on final states with photons can be found in[4].

ATLAS has also studied the possibility to discover GMSB with slepton(s) (co-)NLSP. Again, either the subsequent lepton is prompt, or it can be long lived if $C_{\text {Grav }}>1$. In the first case, it should be well covered by the inclusive analysis described earlier. Otherwise, one should carefully 


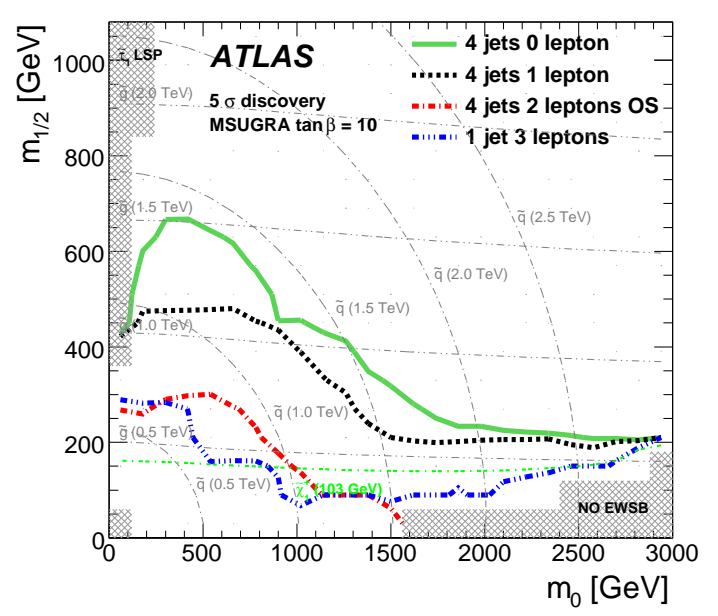

Figure 9. The $1 \mathrm{fb}^{-1} 5 \sigma$ reach contours for the 4-jet plus $E_{T}^{\text {miss }}$ analyses with various lepton requirements for mSUGRA as a function of $\left(m_{0}\right.$ and $\left.m_{1 / 2}\right)$.

study timing and trigger issues. Indeed, at a velocity $(\beta)$ of 1 , muons already only travel $7.5 \mathrm{~m}$ in $25 \mathrm{~ns}$, and with $\beta=0.8$ a slepton takes $15 \mathrm{~ns}$ more than a muon to cross the ATLAS detector. The main issue is to be able to attribute detector signals to the correct beam crossing. ATLAS is working on the implementation of special trigger algorithms to select with high efficiency this type of topologies. This study also applies to the search for R-hadrons in split SUSY.

\subsection{After discovery}

If we eventually discover new physics with jets and $E_{T}^{\text {miss }}$, or with signatures as above such as in the GMSB model, this will not be the end of the story. We will want to know what kind of SUSY we have discovered, and even if it is SUSY at all. In order to be able to answer this question, we have started designing observables and studying their suitability for disentangling the underlying model. The first type of such observable is related to the edges and thresholds in the dilepton, lepton-jet and dijet mass distributions, which would give useful informations on the mass values. Other, more specialized observables, are for

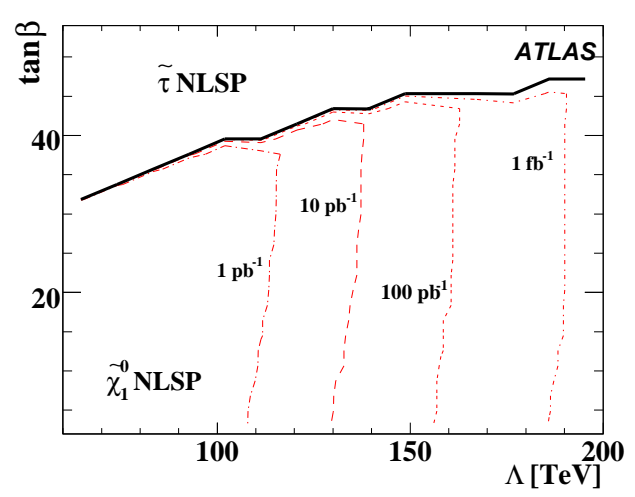

Figure 10. Discovery reach in GMSB parameters $\Lambda$ and $\tan \beta$ in the isolated photon analysis. The value of the other GMSB parameter is $N_{5}=1$, $M_{\text {mess }}=500 \mathrm{TeV}, \mu>0, C_{\text {Grav }}=1$.

instance the rate of tau leptons which hints at the value of $\tan \beta$, or trilepton rates which would tell us about chargino-neutralino couplings. In order to assess SUSY itself, as opposed to models with universal extra dimensions or Little Higgs with Tparity for instance, we will also have to measure the spin of the new particles. Recent ATLAS results on this (reported in [2]) have been produced with full simulation data. This issue in general is discussed in $[5,6]$.

\section{EXOTIC PROCESSES}

In addition to supersymmetry, there are numerous theories beyond the Standard Model (BSM). There are the Grand Unified Theories (GUT), which predict many new particles such as new heavy gauge bosons, neutral or charged $\left(Z^{\prime}, W^{\prime}\right)$, leptoquarks, heavy fermions, doubly charged higgses, etc. There is compositness, which would produce excited states of the fermions $\left(q^{*}, \ell^{*}\right)$, technicolor, Little Higgs or Twin Higgs models, etc. There are also all the various models with extra space-time dimensions, predicting another bulk of new particles or states (Kaluza-Klein excitations of the gauge bosons, graviton, radion, black holes, etc). The list is almost endless and it would be impossible to perform a specific analy- 
sis for all the new particles predicted by the BSM theories. Instead, ATLAS has chosen to concentrate on a number of typical final states, with an inclusive strategy in the same spirit as for SUSY searches. At the time of writing these proceedings, such final state categories are: lepton plus anything ("X"), jet plus X, long lived particles, dibosons and so called "busy events" (the typical final state expected in case of black hole production). Many recent results are reported in [2]; here, only a few of them will be highlighted.

\subsection{Lepton plus $\mathrm{X}$ resonances}

This is the most obvious and easiest final state. The latest ATLAS studies are realistic enough to include for instance the effect of the muon spectrometer misalignment in the computation of the discovery potentials. Figure 11 shows how the $Z^{\prime}$ signal is degraded for several alignment hypotheses (from the ideal one to the most pessimistic). With dilepton final states and taking

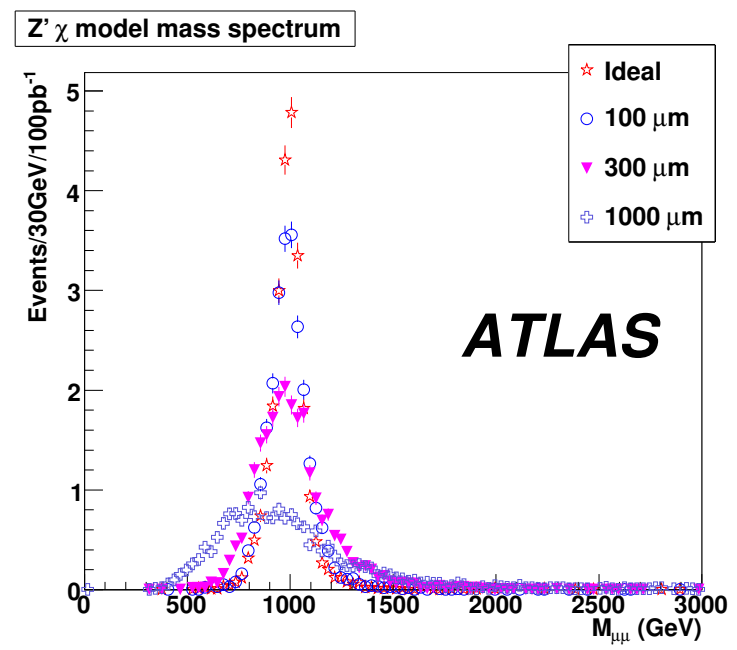

Figure 11. Reconstructed invariant dimuon mass in the $Z_{\chi}^{\prime}$ model for four misalignment scenarios.

the misalignment effect into account, a $Z^{\prime}$ in the $e^{+} e^{-}$or $\mu^{+} \mu^{-}$channel can be discovered up to a mass of about $2 \mathrm{TeV}$ with $1 \mathrm{fb}^{-1}$ of data in the hypothesis of usual GUT models. A degen- erate signal of $\rho_{T}, \omega_{T}$ technimesons in the Technicolor Strawman model could be discovered up to $600 \mathrm{GeV}$ with the same amount of data, and a Randall Sundrum $G^{*}$ (the first excited state of the graviton) between 600 and $1400 \mathrm{GeV}$ for values of $k / M_{P l}$ between 0.005 and 0.025 .

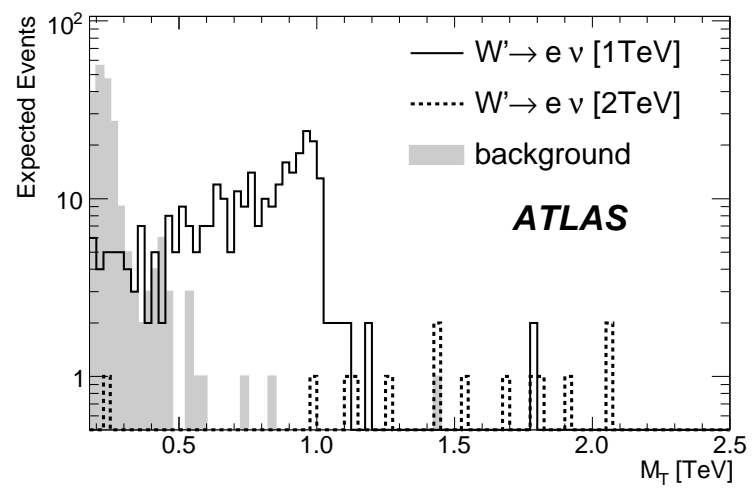

Figure 12. Monte Carlo pseudo experiments showing the transverse mass distribution in the hypothesis of a $W^{\prime}$ in the Sequential Standard Model for $100 \mathrm{pb}^{-1}$.

With lepton plus $E_{T}^{\text {miss }}$ final states, ATLAS can discover a $W^{\prime}$ of at least $3 \mathrm{TeV}$ with $1 \mathrm{fb}^{-1}$ of data. An example of such a signal in the electron channel, with ten times less data, is shown in figure 12 for two mass hypotheses.

With a lepton plus jet final state, ATLAS will discover first and second generation leptoquarks between 300 and $550 \mathrm{GeV}$ for a branching ratio of the leptoquark to lepton plus quark between 0.1 and 1 with only $100 \mathrm{pb}^{-1}$ of good data.

More details on lepton plus $\mathrm{X}$ resonance results can be found in [7].

\subsection{Long lived neutral particles}

Many theories of LHC-scale physics have suggested the possibility of long-lived neutral particles. The Hidden Valley (HV) scenario can serve as a useful setting: it contains many models in which such particles may appear, with a wide range of lifetimes, final states and produc- 
tion mechanisms. In a HV model, to the Standard Model is appended a hidden sector (the "vsector") and a communicator which interacts in both sectors. A barrier (perhaps the communicator's high mass, weak couplings or small mixing angles) weakens the interactions between the two sectors, making production even of light v-sector particles ("v-particles") rare at low energies. At the LHC, by contrast, production of v-particles may be observable; however, the barrier results in a long lifetime for the lightest v-particle.

The study presented here [8] is limited to the Higgs decay $h^{0} \rightarrow \pi_{V}^{0} \pi_{V}^{0}$, where $\pi_{V}^{0}$ is a neutral pseudo-scalar and has a displaced decay mainly to bottom quarks. The following parameters were used to simulate the Higgs decay: $E_{c m}=10 \mathrm{TeV}$, $m_{h^{0}}=140 \mathrm{GeV}, m_{\pi_{V}}=40 \mathrm{GeV}$ and $c \tau=1.5 \mathrm{~m}$. With these parameters approximately $40 \%$ of the decays occur in the ATLAS Inner Detector, $48 \%$ in the calorimeters and the remaining $12 \%$ in the Muon Spectrometer. Clearly in this situation, the main challenge is the trigger. A first naive evaluation of the trigger performance, with standard requirements on jets (at low transverse momentum) and muons, gave efficiencies of the order of 2 to $3 \%$. ATLAS has therefore implemented new, specific, trigger items:

- for events with a decay in the Muon Spectrometer, which show no signal upstream (nor in the Inner Detector, nor in the calorimeters), at least 3 "regions-of interest" ${ }^{3}$ in a $\Delta R=\sqrt{\Delta \eta^{2}+\Delta \phi^{2}}=0.4$ cone will be demanded, in conjunction with the absence of Level-2 jets or tracks in the Inner Detector;

- for events with a decay in the hadronic calorimeter, a Level-2 jet will be demanded, with a transverse energy of more than $35 \mathrm{GeV}$, an energy deposited in the hadronic calorimeter greater than the

\footnotetext{
${ }^{3}$ In the ATLAS trigger system which comprises 3 levels, only the Muon Spectrometer and the calorimeters are used at Level-1; at Level-2, Inner Detector tracks are reconstructed as well, not in the full detector but only in "regions-of interest" defined by signals detected at the first level. A candidate muon is normally rejected at Level-2 if no Inner Detector track can be associated.
}

energy deposited in the electromagnetic calorimeter and no track in the Inner Detector;

- for events with a decay in the Inner Detector, the association of a trackless jet and a muon will be used; this item is still under study for optimization.

The resulting trigger efficiencies and rates are shown on figure 13 for a $10 \mathrm{TeV}$ centre of mass energy and an integrated luminosity of $100 \mathrm{pb}^{-1}$. Timing issues have been checked to be OK, as well as the background: 0.1 to $0.4 \mathrm{~Hz}$ are expected from QCD dijets of $p_{T}=35-70 \mathrm{GeV}$ at an instantaneous luminosity of $10^{31} \mathrm{~cm}^{-2} \mathrm{~s}^{-1}$.

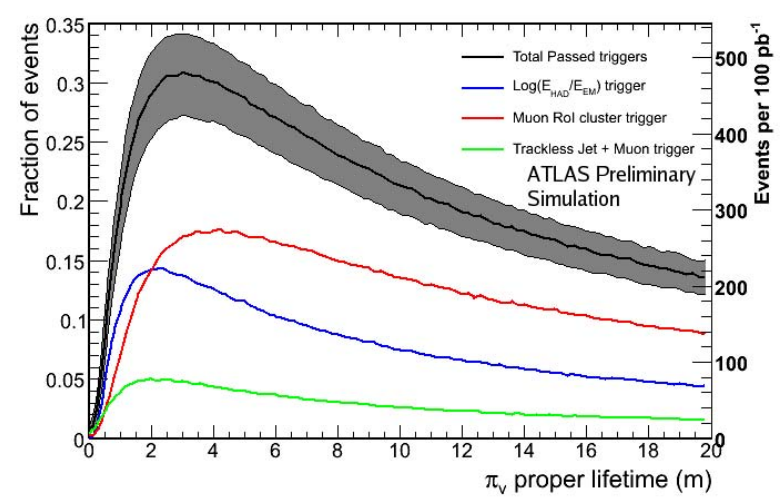

Figure 13. $h^{0} \rightarrow \pi_{V}^{0} \pi_{V}^{0}$ events accepted by longlived particle triggers versus the lifetime assuming $\operatorname{Br}\left(h^{0} \rightarrow \pi_{V}^{0} \pi_{V}^{0}\right)=100 \%$.

\subsection{Signatures of $\mathrm{TeV}$ scale gravity}

With beams of several TeV, LHC is an obvious place to look for $\mathrm{TeV}$ scale gravity. The most studied phenomenon so far is the production of black holes for the case in which the black hole can be treated semi-classically, and is produced and decays according to the concepts of general relativity (GR). The condition required on the mass of gravitational objects for them to be considered GR black holes is given by $M_{B H} \simeq$ $M_{\text {thresh }}=5 M_{D}$, where $M_{D}$ is the fundamental 
Planck scale in higher dimensions. ATLAS has shown that the selection $\sum\left|p_{T}\right|>2.5 \mathrm{TeV}$ (where the sum is over all the particles in the event) and at least one identified lepton with transverse momentum greater than $50 \mathrm{GeV}$ leads to almost no background, and allows to discover black holes with $1 \mathrm{fb}^{-1}$ integrated luminosity up to a black hole mass threshold of about 8 to $9 \mathrm{TeV}$ depending on the number of extra dimensions.

If $M_{D}$ is about $1 \mathrm{TeV}$, imposing the GR condition on black holes leads to a requirement on the black hole mass of $M_{B H} \simeq 5 \mathrm{TeV}$. Thus high mass GR black holes may not be accessible to the LHC. Below this GR threshold we enter the regime of quantum gravity. An exciting possibility for this regime is to treat it in the context of weakly-coupled string theory. It has been shown that highly-excited string states ("string balls") produced below the GR threshold could have a cross section comparable to that of the black hole. Hence, these states will be even more accessible than black holes at the LHC. Their decay is expected to be mainly on the brane, producing multiple jets, leptons and photons, similarly to black holes (hence the "busy events" tag). Using a simple selection [9] $\sum\left|p_{T}\right|+E_{T}^{\text {miss }} \sim 0.8 M_{\text {thresh }}$ and at least one lepton with $p_{T}$ greater than 50 to $100 \mathrm{GeV}$, ATLAS will be sensitive to cross section of at least $185 \mathrm{fb}$. How this can be translated into the parameter of a particular string ball model is shown on figure 14 for a $10 \mathrm{TeV}$ centre of mass energy and an integrated luminosity of $100 \mathrm{pb}^{-1}$.

\section{CONCLUSION}

ATLAS is ready for all sorts of BSM signatures and topologies. With $1 \mathrm{fb}^{-1}$ of integrated luminosity at $14 \mathrm{TeV}^{4}$, ATLAS will be sensitive to a very interesting region of the SUSY parameter space, namely to gluino masses of 500 to $1000 \mathrm{GeV}$. ATLAS will also be sensitive to many other theories of new physics, being able to discover $Z^{\prime}$ up to about $2 \mathrm{TeV}, W^{\prime}$ up to about $3 \mathrm{TeV}, G^{*}$ around $1 \mathrm{TeV} \pm 500 \mathrm{GeV}$, black holes up to a threshold mass of 8-9 $\mathrm{TeV}$.

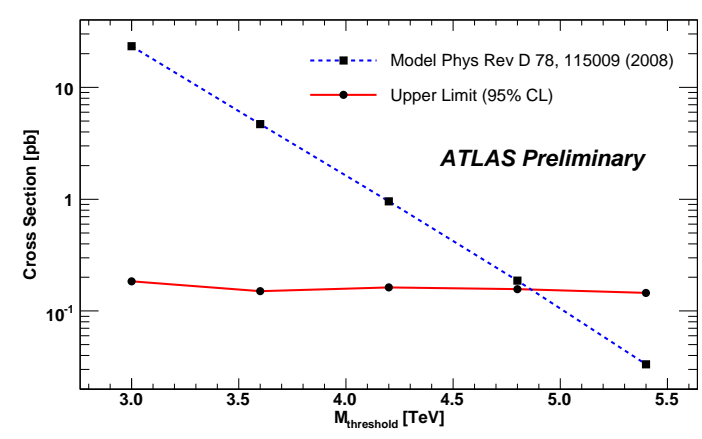

Figure 14. String ball cross section upper limits assuming a luminosity of $100 \mathrm{pb}^{-1}$.

\section{ACKNOWLEDGMENTS}

The author wishes to thank the organizers of the conference, and is also grateful to the conveners of the ATLAS SUSY and Exotics physics groups, Giacomo Polesello and Pierre Savard, for their valuable help.

\section{REFERENCES}

1. The ATLAS Collaboration, "The ATLAS Experiment at the CERN Large Hadron Collider", JINST 3 (2008) S08003.

2. The ATLAS Collaboration, "Expected performance of the ATLAS experiment - Detector, Trigger and Physics", CERN-OPEN2008-020 (2008).

3. The ATLAS Collaboration, "Background estimation for Inclusive SUSY searches - The Tiles Method", ATL-PHYS-PUB-2009-077

4. Haleh Hadavand, these proceedings.

5. Dirk Zerwas, these proceedings.

6. Alan Barr, these proceedings.

7. Luis Flores-Castillo, these proceedings.

8. G. Ciapetti et al., "Triggering on Long-Lived Neutral Particles in the ATLAS Detector", ATL-PHYS-PUB-2009-082.

9. D. Gingrich, "Search for highly-excited string states with ATLAS", ATL-PHYS-PUB-2009011.

${ }^{4} 10 \mathrm{TeV}$ cross sections are typically $50 \%$ lower. 